\title{
USO DE LA TOMOGRAFIA DE EMISION DE POSITRONES EN EL CANCER COLORRECTAL
}

\author{
Dr. Patricio González E, Josefina Jofre M, Teresa Massardo V, Pamela Humeres, José Canessa G, Palunina Sierralta C.
}

Centro PET de Imágenes Moleculares, Medicina Nuclear, Hospital Militar de Santiago.

\begin{abstract}
The value of PET (Positron Emission Tomography) in colorectal cancer is presented. PET is a novel technique that uses F-18-FDG (fluorodeoxiglucose) to assess glucose metabolism by whole body imaging. It has been demonstrated that malignant cells have both increase of glucose uptake and utilization. In colorectal cancer, PET is indicated for staging, assess recurrence, liver metastasis and treatment follow-Up. PET is more sensitive and specific than CT (Computed Tomography) and is cost effective. In $30 \%$ of cases PET may change patient management, avoiding unnecessary procedures.
\end{abstract}

Key words: PET; F-18-FDG (fluorodeoxiglucose), Colorectal cancer.

Resumen: El valor de la tomografía de emisión de positrones (PET) en el cáncer colorrectal es revisado en esta corta comunicación. EI PET es una nueva técnica que emplea F-18-FDG (Fluorodeoxiglucosa) para evaluar el metabolismo glucídico de las células mediante imágenes de todo el cuerpo en un solo examen. Se ha demostrado que las células malignas tienen captación y utilización aumentada de la glucosa. En el cáncer colorrectal el PET esta indicado para el diagnóstico de extensión inicial, sospecha de recurrencia, evaluación de metástasis hepáticas, recidiva tumoral y control de terapias. EI PET es más sensible y específico que la tomografía computada y es una técnica costo efectiva. En aproximadamente un $30 \%$ de los casos ayuda a cambiar el manejo terapéutico de los pacientes evitando procedimientos innecesarios. Este año entrará en fun-

González P. y cols. Uso de la tomografía de emisión de positrones en el cáncer colorectal. Rev Chil Radiol 2002; 8:72-76.

Correspondencia: Dr. Patricio González E.

Casilla 21 La Dehesa. Santiago

ajgonzalez@manquehue.net ciones un ciclotrón en la Comisión Chilena de Energía Nuclear y la primera cámara PET multicristal de alta resolución de Chile y Sudamérica será instalada en el Hospital Militar de Santiago.

Palabras claves: PET; Cáncer Colorrectal; F-18-FDG (fluorodeoxiglucosa).

\section{Introducción}

La tomografía de emisión de positrones (PET), es una técnica de creciente utilización en la práctica clínica, en países donde se encuentra disponible. Se basa en el uso de radiofármacos emisores de positrones, los cuales se unen a moléculas trazadoras de mecanismos fisiopatológicos como el flujo sanguíneo y metabolismo celular. La tabla I nos muestra algunos de estos elementos, siendo el más empleado la Flúor-18 FDG (fluorodeoxiglucosa), que nos informa acerca del metabolismo glucídico y se le emplea en oncología, cardiología y neurología ${ }^{(1-3)}$.

\begin{tabular}{|c|c|}
\hline Trazador & Uso \\
\hline $\begin{array}{l}{ }^{15} \mathrm{O} \\
{ }^{18} \mathrm{FDG} \\
{ }^{18} \mathrm{FDOPA} \\
\mathrm{H}_{2}{ }^{15} \mathrm{O} \\
\mathrm{C}-11 \text { metionina } \\
\mathrm{C}-11 \text { acetato } \\
\mathrm{C}-11 \text { colina } \\
{ }^{13} \mathrm{NH}_{3}\end{array}$ & $\begin{array}{l}\text { Cardiología } \\
\text { Oncología } \\
\text { Neurología } \\
\text { Cardiología } \\
\text { Oncología } \\
\text { Cardiología } \\
\text { Oncología } \\
\text { Cardiología }\end{array}$ \\
\hline
\end{tabular}

El F-18 es producido en ciclotrón, permite marcar la FDG, la cual una vez inyectada al paciente, es detectada mediante una cámara de positrones, obteniendo imágenes de la distribución de este 
radiotrazador en todo el organismo. EI PET se utiliza mayoritariamente en oncología que comprende prácticamente el $90 \%$ de los exámenes. Las otras aplicaciones abarcan en conjunto el $10 \%$ restante.

En tumores se ha demostrado un aumento en la utilización de la glucosa, lo cual además se encuentra en relación con su grado de malignidad. Este hecho permite localizar neoplasias primarias y sus metástasis con adecuada sensibilidad y especificidad.

Se ha estudiado una amplia variedad de tumores, pero ha parecido de interés elegir el cáncer colorectal para ilustrar el valor de esta nueva tecnología.

En nuestro país entrará este año en funciones un ciclotrón en la Comisión Chilena de Energía Nuclear y la primera cámara PET multicristal de alta resolución de Chile y Sudamérica será instalada en el Hospital Militar de Santiago.

\section{Indicaciones}

Las indicaciones actuales de la tomografía PET en el cáncer colorectal son las siguientes:

1. Diagnóstico de extensión inicial

2. Sospecha de recurrencia o recidiva tumoral

3. Estudios de re-estadificación, previo a tratamiento quirúrgico

4. Evaluación de metástasis hepáticas

5. Control de terapias: quimioterapia, radioterapia

\section{Casos ilustrativos}

Ilustraremos con algunos ejemplos y experiencias de estas indicaciones.

Diagnóstico inicial: La figura 1 nos muestra imágenes de PET en un paciente que evidenció intensa captación de glucosa en un tumor primario de colon transverso, sin lesiones de extensión. El valor en este caso es estadificar correctamente al paciente, evitando procedimientos innecesarios. Sin embargo, el estudio de PET no se utiliza para el diagnóstico de la lesión primaria, el cual se hace mediante

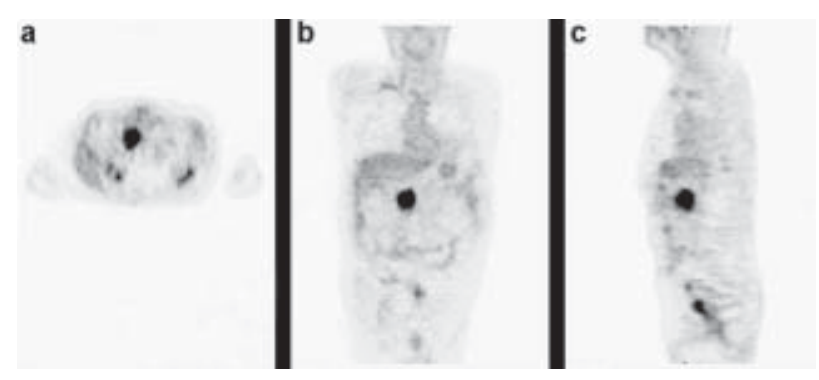

Figura 1. a,b,c PET con F-18-FDG. Cortes axial (a), coronal (b) y sagital (c), explorando el tronco en su totalidad. Se evidencia intensa captación de glucosa en tumor primario ubicado en colon transverso colonoscopía y biopsia o enema baritado. Debe recordarse que las lesiones benignas tienen nula 0 escasa captación de la F-18-FDG.

Recurrencia: Respecto a recurrencia de enfermedad, luego del tratamiento inicial, los pacientes son evaluados mediante marcadores tumorales, destacando el CEA (antígeno carcinoembrionario) y el CA19.9. Ante aumento de estos, se realiza exámenes complementarios que incluyen: colonoscopía, tomografía axial computada (TAC), ultrasonografía, eventualmente resonancia magnética (RM), radiografía de tórax y laparoscopía. En aquellos casos en que esto es posible se incluye biopsia. Si estos estudios resultan negativos, entonces puede realizarse control del paciente en 3-6 meses.

Hay que hacer notar que aproximadamente un 30 a $40 \%$ de los pacientes portadores de cáncer colorectal, experimentan recurrencia a los cinco años. La localización preferencial es el hígado.

La sensibilidad para detectar recurrencias en el caso de el PET es de un $93 \%$, la TAC tiene un $71 \%$ y en el marcador tumoral (CEA) es de un $59 \%$ solamente. Respecto a la especificidad esta es de $96 \%$ para el PET, $89 \%$ para la TAC y $84 \%$ para el CEA. Estos datos fueron obtenidos en 1387 pacientes en los cuales se realizaron 2244 exámenes $^{(3)}$.

Valk y col. ${ }^{(5)}$ en 1999 publicaron, la sensibilidad para detectar recurrencia por territorios encontrando la siguiente distribución: a nivel del hígado 95\% para el PET y un $84 \%$ para la TAC. En pelvis de $97 \%$ para el PET y $68 \%$ para la TAC. En el abdomen en general de $79 \%$ para el PET y $46 \%$ para la TAC. En el retroperitoneo la sensibilidad de el PET fue de 100\% y en el caso de TAC sólo un $58 \%$.

Flanagan y col..$^{(6)}$ en 1998, sobre la base de 22 pacientes en quienes se había realizado tamizaje de recidiva mediante CEA, que resultó positivo con estudio convencional negativo, encontró un valor predictivo positivo de $89 \%$ (15/17) y un valor predictivo negativo de $100 \%(5 / 5)$ para el PET.

La figura 2 ilustra un estudio de recidiva en cáncer colorectal, demostrando múltiples zonas de captación de la glucosa radioactiva marcada con Flúor18 en región supraclavicular, cadenas lumbo-aórticas especialmente izquierda, en campos pulmonares y en región pelviana izquierda.

Estadificación: La asociación de un PET con marcadores tumorales elevados puede ayudar al manejo del paciente. Simo, en un estudio de 93 pacientes que tenían CA elevado, y en los cuales se realizó un $P E T$, demuestra que 64 de éstos resultaron positi$\operatorname{vos}^{(7)}$. De este grupo, 39 tenían lesiones únicas que fueron a cirugía y por el contrario 25 tenían una enfermedad diseminada por lo tanto se sometieron solamente a quimioterapia. En los que el PET fue negativo, en total 29 casos, se hizo seguimiento de 17 meses demostrando que 25 de ellos estaban li- 

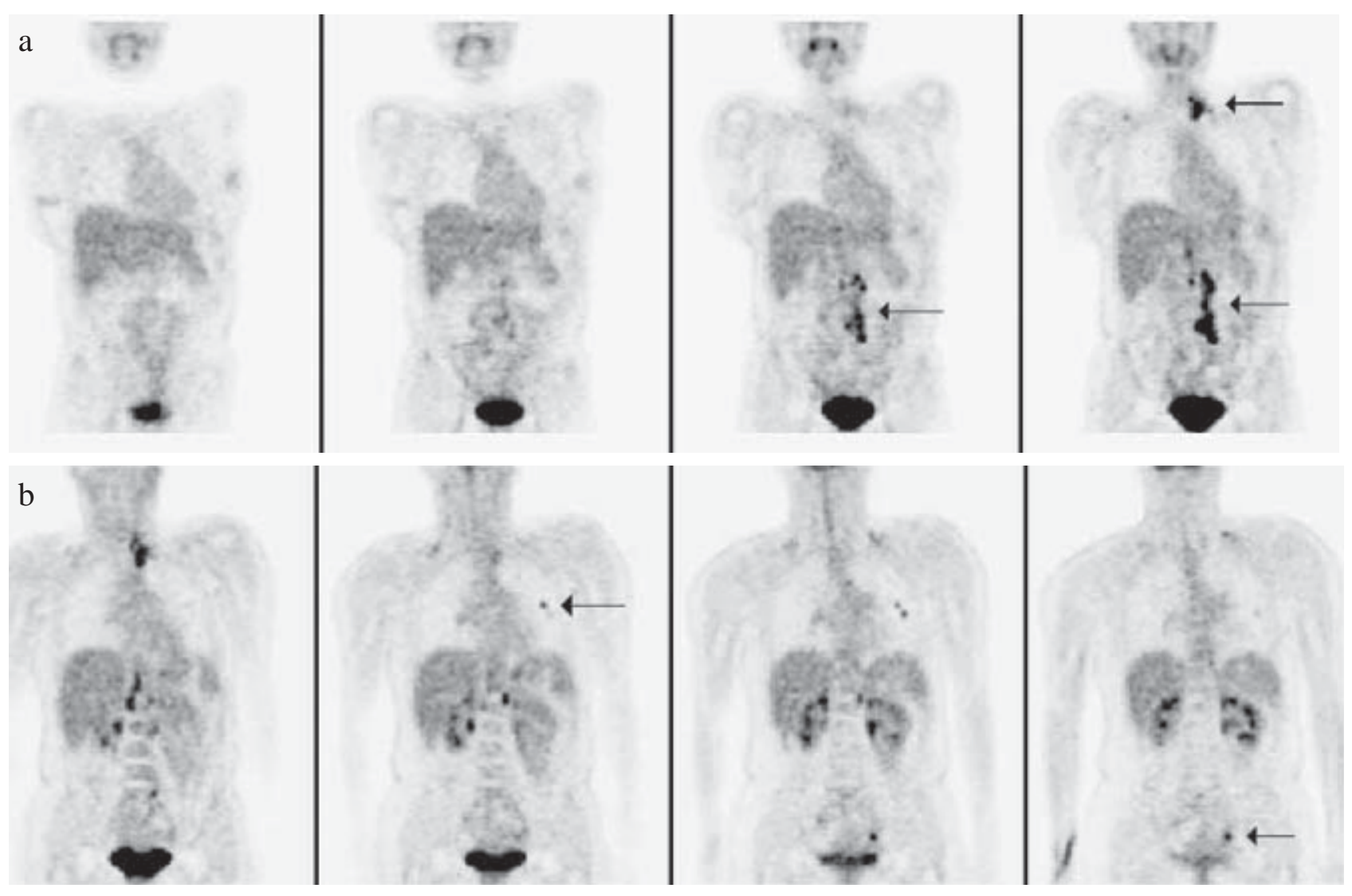

Figura 2. a,b. PET con F-18-FDG. Demuestra en cortes coronales un estudio de recidiva en cáncer colorectal. Las flechas marcan múltiples zonas de captación de la glucosa radioactiva marcada con Flúor-18 en región supraclavicular, cadenas lumbo-aórticas especialmente izquierda (a), en campos pulmonares y en región pelviana izquierda (b).

bres de enfermedad y en sólo cuatro se presentaron áreas de recidiva, dos peritoneales, uno en hígado y otra pélvica. Los casos en que la tomografía de positrones fue negativa correspondieron a lesiones muy pequeñas o de carácter difuso en el peritoneo.

La figura 3 nos muestra las imágenes de un paciente en evaluación de recurrencia y se compara con RM. La vejiga da una imagen de captación intensa hacia anterior, lo cual es habitual por eliminación de la F18-FDG por el riñón.

Es de interés el estudio de Valk y col.(5) en 78

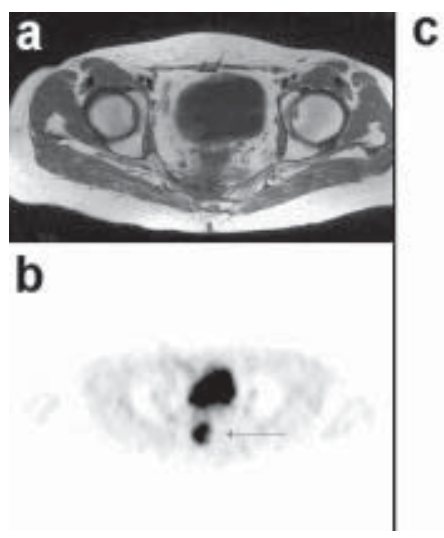

pacientes que tenían cáncer colorectal con lesión única aparente determinado por TAC. EI PET demostró que en 25 de estos casos (32\%) había una diseminación múltiple y cuatro casos (8\%) que la TAC era falsamente positiva. En conclusión estos son 29 pacientes $(40 \%)$ que estaban mal estadificados según la TAC y habrían sido sometidos a cirugía innecesaria.

Respuesta a terapia: Otro aspecto interesante de analizar es el valor potencial del PET para evaluar la respuesta a la terapia. Esto se ve favorecido porque en general los cambios metabólicos preceden a los morfológicos. También resulta ventajoso contar con estudios de cuantificación ya sea absoluta o relativa que son susceptibles de realizar mediante la tomografía PET. Así, es factible medir flujo sanguíneo o tasas de consumo absoluto de metabolitos. En

Figura 3. a,b,c. Evaluación de recurrencia de carcinoma colorectal PET F-18-FDG y RM. La resonancia magnética (a) mostró una lesión muy sospechosa en la región presacra. La tomografía PET muestra intensa captación (flechas). (b), Corte axial y (c) corte sagital. La imagen de captación más intensa hacia anterior es la vejiga, habitual de visualizar por eliminación renal de la $F^{18}-F D G$. 
la práctica, resulta simple y rápido de emplear el valor estandarizado de captación (SUV), que relaciona la fracción de dosis inyectada presente en el tumor o sus metástasis.

Distintos autores como Findlay y col. ${ }^{(8)}$ y Haberkorn y col. ${ }^{(9)}$ han estudiado la respuesta a tratamiento quimioterápico o radioterápico en cáncer colorectal. El primero encuentra en 18 pacientes que la cuantificación de la captación de la lesión en el hígado, permite identificar aquellos pacientes respondedores a la quimioterapia. El segundo estudia 49 pacientes sometidos a radioterapia y concluye que los controles de PET deben hacerse 6 meses después de la terapia para evitar cambios inflamatorios como causa de captación de F-18-FDG. Concluye además que el PET es más sensible que el CEA para detectar recurrencia.

La figura 4 nos muestra un paciente con cáncer colorectal con lesiones metastásicas en hígado, y una buena respuesta después de la quimioterapia y la figura 5 ilustra el caso de una mala respuesta a esta.

Distintos autores detallados en la tabla II han estudiado el valor del PET en el cáncer colorectal y han demostrado un $29 \%$ promedio de cambios en el manejo en 349 pacientes.

Figura 4. a,b Paciente con cáncer colorectal con lesiones metastásicas en hígado, que experimenta una favorable evolución con disminución de las lesiones después de la quimioterapia (flechas).

Tabla II. Cambio en el manejo terapéutico (\%)

$\begin{array}{lcc}\text { Autor } & \text { No pacientes }^{\circ} & \text { Cambio manejo (\%) } \\ \text { Beets } & 35 & 40 \%(14 / 35) \\ \text { Vitola } & 24 & 25 \%(6 / 24) \\ \text { Lai } & 34 & 29 \%(10 / 34) \\ \text { Delbeke } & 52 & 33 \%(17 / 52) \\ \text { Ogunbiyi } & 23 & 44 \%(10 / 23) \\ \text { Valk } & 78 & 31 \%(24 / 78) \\ \text { Flamen } & 103 & 20 \%(21 / 103) \\ & & 29 \%(102 / 349) \\ \text { Total } & 349 & \end{array}$

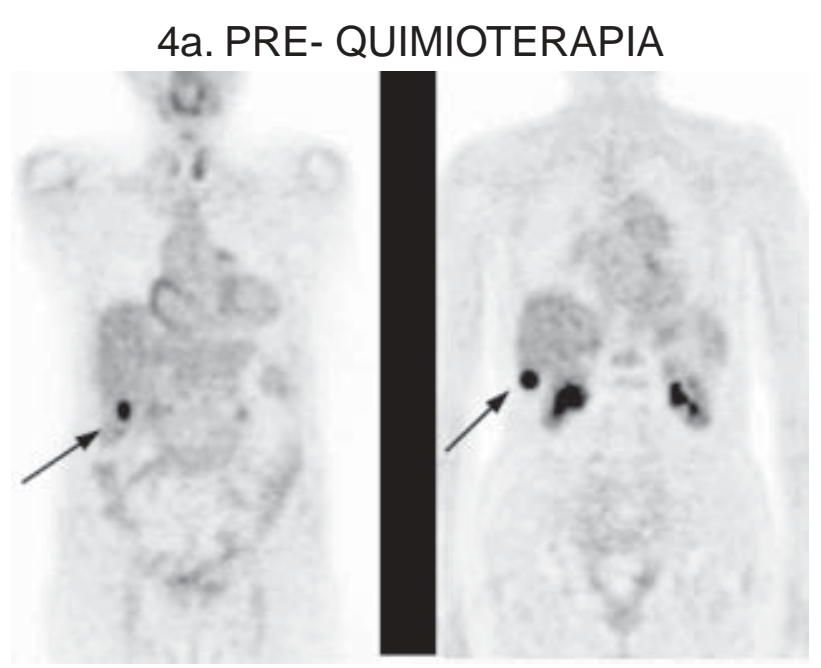

4b. POST- QUIMIOTERAPIA

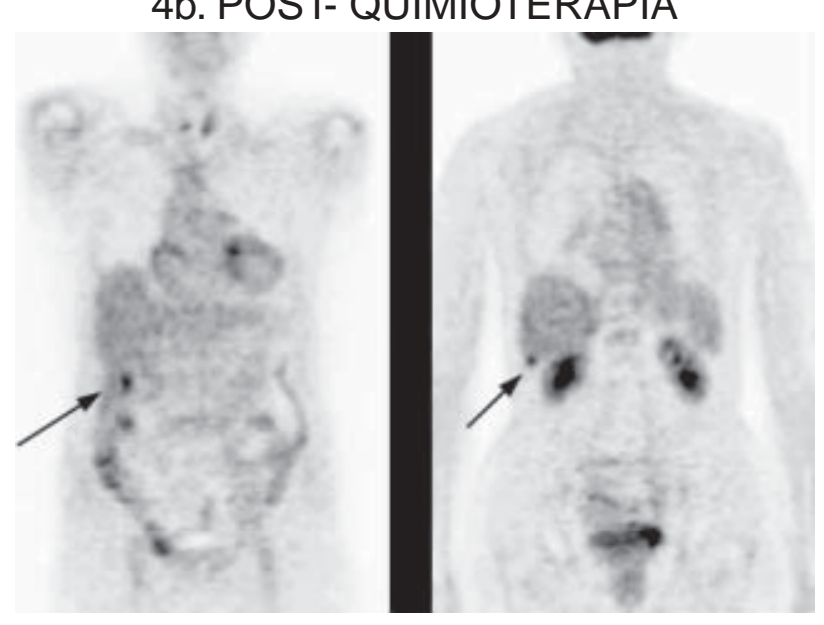

\section{Conclusión}

EI PET para cáncer colorectal posee varias ventajas. En primer lugar es una exploración de cuerpo entero por lo que su evaluación es más certera. Además se ha demostrado por varios autores que tiene muy buena sensibilidad para detección de recurrencia tumoral. Asimismo, puede tener impacto en el manejo terapéutico, en la relación costo-efectividad y también en el manejo del tratamiento, evitando procedimientos innecesarios.

Debe sin embargo, mantenerse cautela en algunos casos ya que hay lesiones pequeñas o difusas que pueden no ser detectadas por esta tecnología.

\section{Agradecimientos}

Al Dr. Ignasi Carrio y Dr Marc Simo, por el apoyo para conseguir los casos clínicos en el Centro de PET Cetir de Barcelona, España, durante estadía de entrenamiento del autor pricipal del artículo, en 2002. 


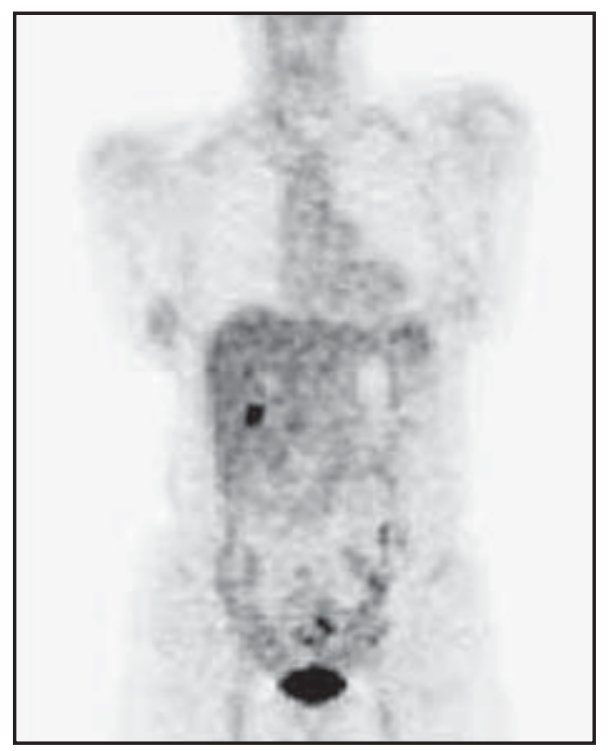

a. PRE- QUIMIOTERAPIA
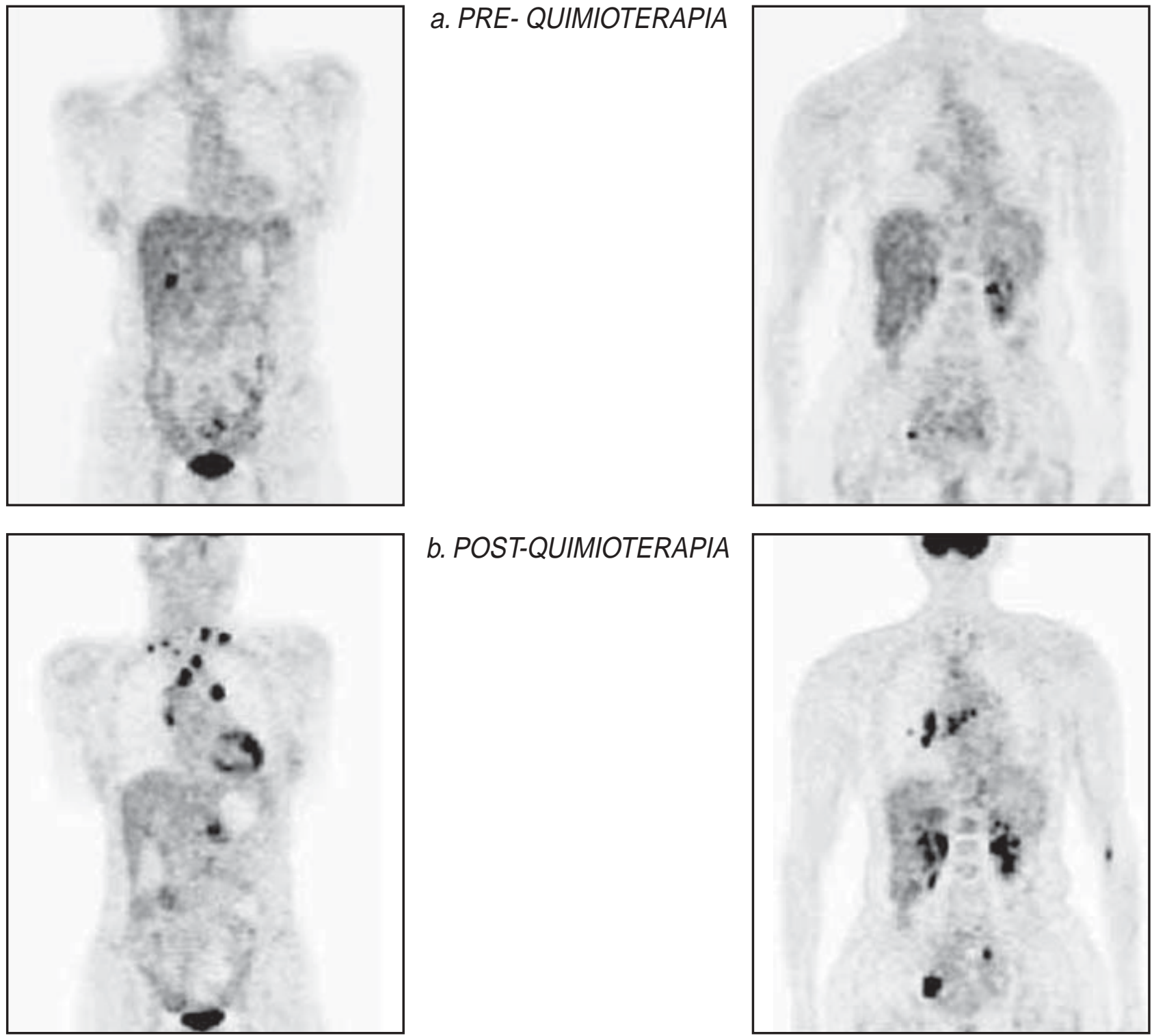

\section{b. POST-QUIMIOTERAPIA}

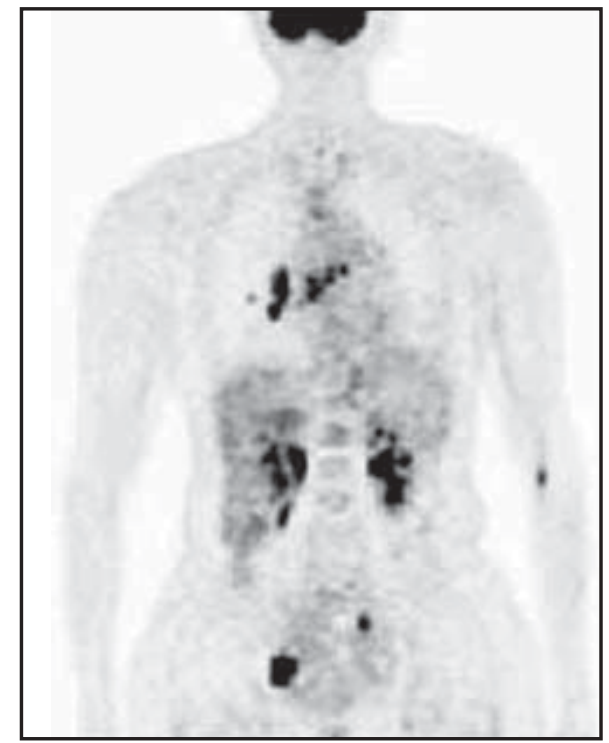

Figura 5. a,b Paciente de 56 años que presenta un antígeno CEA elevado. El PET detecta una lesión metastásica en hígado y algunas lesiones en linfonodos de cadenas lumboaorticas así como en región ilíaca derecha (a). No hubo respuesta a la quimioterapia y en el PET de control se puede observar que han aumentado notoriamente los focos de captación de glucosa en cadena lumboaortica bilateral, en mediastino y asimismo en la región ilíaca y pelviana (b).

\section{Bibliografía}

1. González P, Massardo V, Alavi A. Valores estandarizados de captación miocárdica (SUV) en estudios con PET F18-FDG en pacientes oncológicos. Rev Chil Cancerol Hematol. 2000; 10: 109

2. Delbeke D. Oncological Applications of $F^{18}$-FDG PET Imaging: Brain Tumors, Colorectal Cancer, Lymphoma and Melanoma. J Nucl Med. 1999; 40: 591-603

3. Gambhir S.S, Czernin J, Schwimmer J, y col. A Tabulated Summary of the $\mathrm{F}^{18}$-FDG PET Literature. J Nucl Med. 2001; 42: 1S-93S

4. Valk PE, Pounds TR, Tesar RD y col. Cost-effectiveness of PET imaging in clinical oncology. Nucl Med Biol. 1996;23:737-43

5. Valk PE, Abella-Columna E, Haseman MK y col. Wholebody PET imaging with $\left[\mathrm{F}^{18}\right]$ fluorodeoxyglucose in management of recurrent colorectal cancer. Arch Surg 1999;134:503-11

6. Flanagan FL, Dehdashti F, Ogunbiyi OA y col. Utility of FDG-PET for investigating unexplained plasma CEA elevation in patients with colorectal cancer. Ann Surg 1998; 227: 319-23

7. Simo M. Tomografia por Emision de Positrones en el Cancer Colorrectal. Capítulo en: Medicina Nuclear. Aplicaciones Clínicas. I Carrio y P González, Masson, Barcelona, España. En Prensa.

8. Findlay $\mathrm{M}$, Young $\mathrm{H}$, Cunningham D. Y col. Noninvasive monitoring of tumor metabolism using fluorodeoxyglucose and positron emission tomography in colorectal cancer liver metastases: correlation with tumor response to fluorouracil. J Clin Oncol 1996; 14:700-8

9. Haberkorn U, Strauss LG, Dimitrakopoulou A. y col. PET studies of fluorodeoxyglucose metabolism in patients with recurrent colorectal tumors receiving radiotherapy. J Nucl Med 1991; 32:1485-90. 